\title{
Mycoplasma pneumoniae as a causative agent of community-acquired pneumonia in children: clinical features and laboratory diagnosis
}

Biljana Medjo ${ }^{1,2^{*}}$, Marina Atanaskovic-Markovic ${ }^{1,2}$, Snezana Radic ${ }^{3}$, Dimitrije Nikolic ${ }^{1,2}$, Marija Lukac ${ }^{1,2}$ and Slobodanka Djukic ${ }^{4}$

\begin{abstract}
Background: Mycoplasma pneumoniae is a common cause of community-acquired pneumonia (CAP) in children. The aim of this study was to assess the prevalence of Mycoplasma pneumoniae infection in children with CAP and find clinical, radiological and laboratory features helpful to diagnose Mycoplasma pneumoniae pneumonia. Furthermore, we evaluated the value of serology, real-time PCR (RT-PCR) and culture for the accurate diagnosis of Mycoplasma pneumoniae pneumonia.

Methods: The study included 166 children aged between 1 and 15 years with radiologically confirmed pneumonia. Throat swab specimens were cultured and assessed by RT-PCR for the presence of Mycoplasma pneumoniae. Mycoplasma pneumoniae-specific lgM and IgG antibodies were determined using ELISA in paired sera.

Results: Mycoplasma pneumoniae pneumonia was diagnosed in $14.5 \%$ CAP cases. Cough ( $p=0.029$ ), headache ( $p=0.001)$ and wheezing $(p=0.036)$ were more frequent in children with Mycoplasma pneumoniae pneumonia compared to children with pneumonia caused by other pathogens. Logistic regression analysis showed that headache (odds ratio $[O R]=36.077, p=0.001)$ and wheezing $(\mathrm{OR}=5.681, \mathrm{p}=0.003)$ were significantly associated with MP pneumonia. Neither radiological findings, nor common laboratory parameters distinguished Mycoplasma pneumoniae infection in children with CAP. Using IgG serology in paired sera as the gold standard, we found that sensitivity of IgM serology, RT-PCR and culture was equal (81.82\%), while specificity values were 100\%, $98.6 \%$ and $100 \%$ respectively. We observed that combination of IgM detection in acute-phase serum and RT-PCR was positive for $91.7 \%$ of cases with Mycoplasma pneumoniae infection.

Conclusions: There are no characteristic radiological findings, or routine laboratory tests that would distinguish CAP caused by Mycoplasma pneumoniae from other CAP. It was found that clinical features such as headache and wheezing are indicative for Mycoplasma pneumoniae infection. Furthermore, it was found that during the acute phase of disease, detection of IgM antibodies in combination with RT-PCR allows for precise and reliable diagnosis of Mycoplasma pneumoniae infections in children.
\end{abstract}

Keywords: Children, Community-acquired pneumonia, Diagnosis, Mycoplasma pneumoniae, Polymerase chain reaction

\footnotetext{
* Correspondence: medjo.biljana@gmail.com

'Medical Faculty, University of Belgrade, Dr Subotica 8, 11000 Belgrade,

Serbia

2University Children s Hospital, Tirsova 10, Belgrade, Serbia

Full list of author information is available at the end of the article
}

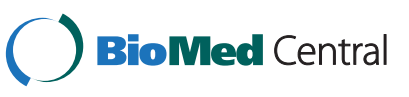

2014 Medjo et al.; licensee BioMed Central. This is an Open Access article distributed under the terms of the Creative Commons Attribution License (http://creativecommons.org/licenses/by/4.0), which permits unrestricted use, distribution, and reproduction in any medium, provided the original work is properly credited. The Creative Commons Public Domain Dedication waiver (http://creativecommons.org/publicdomain/zero/1.0/) applies to the data made available in this article, unless otherwise stated. 


\section{Background}

Mycoplasma pneumoniae (MP) causes up to $40 \%$ of community-acquired pneumonia (CAP) in children and about $18 \%$ of infections in patients requiring hospitalization [1]. In the majority of cases of suspected MP pneumonia, the presumptive diagnosis is made on clinical and radiological findings alone. However, MP is not susceptible to the $\beta$-lactam antibiotics regularly used for the treatment of pediatric pneumonia [2], therefore a specific diagnostic tool is extremely important. Laboratory diagnosis of MP infection in clinical practice have been based on serology, culture and polymerase chain reaction (PCR), although each of them has specific limitations [3].

In Serbia, however, diagnosis of MP infection is most often based on serology. Consequently, the precise incidence of MP infection in children with CAP remains unknown. Therefore, we conducted a study to assess the incidence of MP pneumonia, define clinical, radiological and laboratory features specific for MP pneumonia and to evaluate the value of serology, real-time PCR (RT-PCR) and culture for the accurate diagnosis of MP pneumonia.

\section{Methods}

\section{Study population}

Previously healthy children aged between 1 and 15 years with symptoms, signs and chest radiographs consistent with community-acquired pneumonia examined in the Emergency department of University Childrens Hospital in Belgrade from April 2012 to March 2014 were prospectively enrolled in this study. Pneumonia was defined as the presence of fever, acute respiratory symptoms (cough, tachypnoea, difficult breathing) or both, plus presence of new infiltrate on chest radiography or consolidation not attributable to some other aetiology [4]. Children who received anti-mycoplasma therapy were excluded from the study. This study was approved by Ethics Committee of University Children s Hospital in Belgrade. Patients were enrolled in study after written informed consent had been obtained from their parents or guardians.

Demographic and clinical data were collected uniformly from all children who were included in the study. Chest radiographs were taken and reviewed by pediatric radiologist. Radiological findings were classified as interstitial infiltration, linear opacities, patchy infiltration, segmental or lobar consolidation, reticulo-nodular infiltrate or pleural effusion [5]. Throat swabs taken at enrolment were cultured and assessed by RT-PCR. Blood samples were obtained at the time of enrolment and two to four weeks after. White blood cell (WBC) count, $\mathrm{C}$-reactive protein (CRP) and serological tests were performed at enrolment. The patients were hospitalized on the basis of the clinical decision of the pediatricians on duty. Data regarding clinical course of illness were also recorded.

\section{Culture methods}

Clinical specimens were inoculated on PPLO (Pleuro pneumonia like organisms) broth and agar bases (HiMedia, HiMedia Laboratories, Mumbai, India) supplemented with Mycoplasma supplements (HiMedia) and glucose $(0.1 \%)$ (HiMedia). The cultures were incubated at $37 \mathrm{C}$ in an atmosphere of $5 \% \mathrm{CO}_{2}$ and $95 \%$ air. The broth cultures were observed daily for 21 days and the growth of MP is indicated by phenol red (acid production because of glucose degradation leads to a yellowing of the broth). The agar cultures were examined microscopically after 21 days of incubation for the presence of typical colonies (the isolated colonies were identified by their characteristic homogeneous granular appearance and stained with Dienes staining).

\section{DNA extraction}

The Qiagen QIAamp DNA minikit (QIAGEN, Hilden, Germany) was used for nucleic acid extraction from examined respiratory specimens according to the manufacturer s instructions. The elution volume was reduced to $30 \mu \mathrm{l}$.

\section{Real-time PCR}

Each nucleic acid extract from examined respiratory specimens was tested with our in-house real-time PCR assay. This method, targeting a 125-bp fragment of the M. pneumoniae P1 cytadhesin gene, was performed using the primers P1-204 [5'-GTGAACGTATCGTAACACGAGCT TT-3'), P1-328 (5'-TGGTTTGTTGACTGCCACTGC$3^{\prime}$ ], the TaqMan probe P1-284R [5'-TAM-TTGTCGCGC ACTAAGGCCCACG-MGB-3'], and a commercial internal control (IC), the TaqMan exogenous internal positive control (VIC dye) (Applied Biosystems, Foster City, USA). Real-time PCR was performed in $25 \mu \mathrm{l}$ of a reaction mixture consisting of $12.5 \mu \mathrm{l}$ of $2 \mathrm{X}$ TaqMan universal PCR master mix (Applied Biosystems, Foster City, USA), $0.9 \mu \mathrm{M}$ of each primer, $0.25 \mu \mathrm{M}$ of probe, $0.5 \mu \mathrm{l}$ of IC DNA, $2.5 \mu \mathrm{l}$ of IC primers/probe, and $5 \mu \mathrm{l}$ of extracted DNA. Amplifications were performed using a Applied Biosystems 7500 Real-Time PCR system (Applied Biosystems, Foster City, USA) under the following conditions: $2 \mathrm{~min}$ at $50 \mathrm{C}$ and $10 \mathrm{~min}$ at $95 \mathrm{C}$, followed by 45 cycles of $15 \mathrm{~s}$ at $95 \mathrm{C}$ and $1 \mathrm{~min}$ at $60 \mathrm{C}$.

\section{Serological testing}

Serum was separated from the venous blood samples and stored at $-20 \mathrm{C}$ till assayed. For detection of MP antibodies, a commercially available IgM and an IgG enzyme-linked immunosorbent assay (ELISA Euroimmun, Lbeck, Germany) were performed. The serological diagnosis was defined when IgM antibodies were detected $(>1.1)$ in single serum or if at least 4-fold increase in the IgG antibody titer was detected in the paired sera. 


\section{Definition of acute MP infection}

Acute MP infection was defined if at least one method was positive among detection of MP by culture or by PCR or detection of MP-specific antibodies by serology.

\section{Statistical analysis}

Clinical data of the separate groups were described my mean values and standard deviations or percentages, according to the type of variable. Students $t$-test and ManneWhitney $U$-test were used to compare continuous variables between the group and Chi-square test and Fishers exact test were used for categorical variables. Furthermore, the variables resulting significant at the univariate analysis were subjected to logistic regression and odds ratios and their 95\% confidence interval (CI) were calculated. A p value of $<0.05$ was considered statistically significant. Sensitivity and specificity were calculated using SS calculation comparing truly/falsely positive/negative with frequencies of referent methods (golden standard).

\section{Results}

Current study included 166 children with CAP. Among them $86(51.8 \%)$ were males and 80 (48.2\%) were females $(\mathrm{p}=0.641)$. The mean age of the study population was 6.354 .52 years, 88 (53.0\%) children were less than 5 years old, while $78(47.0 \%)$ were 515 years old $(\mathrm{p}=0.438)$. Mycoplasma pneumoniae pneumonia was diagnosed in 24 (14.5\%) children according to our diagnostic criteria, with significant difference in the age distribution, $25 \%$ of them were below 5 years and $75 \%$ were 5 years and over $(\mathrm{p}=0.003)$. Mycoplasma pneumoniae infections were detected throughout the year. A highest proportion of MP infections was recorded in the autumn (33.3\%), followed by $29.2 \%$ in winter, $25 \%$ in spring and $12.5 \%$ in summer but the difference was not statistically significant $(\mathrm{p}=0.506)$.

In our study MP infection was present in boys more often than in girls $(\mathrm{p}=0.014)$. Children with MP pneumonia were significantly older $(\mathrm{p}=0.001)$, and had longer duration of fever $(p=0.021)$ and cough before enrolment $(\mathrm{p}=0.026)$ compared to children with non-MP pneumonia (Table 1).

Clinical characteristics of children in both groups were further analyzed and compared. We found that cough $(\mathrm{p}=0.029)$, headache $(\mathrm{p}=0.001)$ and wheezing $(\mathrm{p}=0.036)$ were more frequent in children with MP pneumonia compared to children with non-MP pneumonia (Table 2).

We did not observe other significant differences regarding symptoms, signs or physical findings between these two groups (Table 2). The variables found to be more frequent in children with MP pneumonia were further analyzed by logistic regression. It was found that headache (odds ratio $[\mathrm{OR}]=36.077,95 \%$ confidence
Table 1 Comparison of the demographic and clinical data between children with Mycoplasma pneumoniae pneumonia and in children with pneumonia caused by other pathogens at enrolment

\begin{tabular}{|c|c|c|c|}
\hline & $\begin{array}{l}\text { Mycoplasma } \\
\text { pneumoniae } \\
\text { pneumonia } \\
(n=24)\end{array}$ & $\begin{array}{l}\text { Non-Mycoplasma } \\
\text { pneumoniae } \\
\text { pneumonia } \\
(n=142)\end{array}$ & $P$ value \\
\hline \multicolumn{4}{|l|}{$\operatorname{Sex}(\%)$} \\
\hline Males & 75 & 47.9 & $p=0.014$ \\
\hline Females & 25 & 52.1 & \\
\hline Age (years) & 9.895 .21 & 5.754 .13 & $p=0.001$ \\
\hline $\begin{array}{l}\text { Duration of symptoms } \\
\text { before enrolment (days) }\end{array}$ & 7.334 .89 & 5.753 .78 & $p=0.089$ \\
\hline $\begin{array}{l}\text { Fever duration before } \\
\text { enrolment (days) }\end{array}$ & 5.503 .65 & 3.832 .93 & $p=0.021$ \\
\hline $\begin{array}{l}\text { Cough duration before } \\
\text { enrolment (days) }\end{array}$ & 7.174 .89 & 5.014 .25 & $p=0.026$ \\
\hline
\end{tabular}

Date are presented as mean SD unless otherwise indicated.

interval $[\mathrm{CI}]=4.897-265.811, \mathrm{p}=0.001)$, wheezing $(\mathrm{OR}=5.681$, 95\% $\mathrm{CI}=1.776-18.175, \mathrm{p}=0.003)$, mail gender $(\mathrm{OR}=0.162$, $95 \% \mathrm{CI}=0.048-0.548, \mathrm{p}=0.003)$ and age 5 years and older $(\mathrm{OR}=3.067,95 \% \mathrm{CI}=1.016-9.251, \mathrm{p}=0.047)$ were significantly associated with MP pneumonia.

The radiological findings in children with MP pneumonia were variable and summarized in Table 3 . No significant difference was observed in relation to the radiological findings in the children with MP pneumonia and those with pneumonia caused by other pathogens. We noticed that unilateral lung involvements were more often than bilateral in children with and without MP infection $(83.3 \%$ vs. $16.7 \%, 70.4 \%$ vs. $29.59 \%, p=0.191$

Table 2 Comparison of the symptoms, signs and physical findings between children with Mycoplasma pneumoniae pneumonia and in children with pneumonia caused by other pathogens at inclusion

\begin{tabular}{llll}
\hline & $\begin{array}{l}\text { Mycoplasma } \\
\text { pneumoniae } \\
\text { pneumonia } \\
\text { (n=24) }\end{array}$ & $\begin{array}{l}\text { Non - Mycoplasma } \\
\text { pneumoniae } \\
\text { pneumonia } \\
\text { (n=142) }\end{array}$ & P value \\
\hline Fever & $20(83.3)$ & $132(93.0)$ & \\
Cough & $24(100.0)$ & $118(83.1)$ & $\mathrm{p}=0.117$ \\
Coryza & $4(16.7)$ & $36(25.3)$ & $\mathrm{p}=0.029$ \\
Difficulty breathing & $2(8.3)$ & $16(11.3)$ & $\mathrm{p}=0.357$ \\
Headache & $6(25.0)$ & $2(1.4)$ & $\mathrm{p}=0.669$ \\
Vomiting & $4(16.7)$ & $30(21.1)$ & $\mathrm{p}=0.001$ \\
Tachypnoea & $4(16.7)$ & $32(22.5)$ & $\mathrm{p}=0.617$ \\
Rales & $8(33.3)$ & $40(28.1)$ & $\mathrm{p}=0.649$ \\
Wheezing & $8(33.3)$ & $22(15.5)$ & $\mathrm{p}=0.606$ \\
Atopy or asthma & $12(50.0)$ & $60(42.3)$ & $\mathrm{p}=0.036$ \\
\hline
\end{tabular}

Date are presented as number (\%). 
Table 3 Comparison of the radiological characteristics and laboratory data between children with Mycoplasma pneumoniae pneumonia and in children with pneumonia caused by other pathogens

\begin{tabular}{ccc}
\hline & $\begin{array}{l}\text { Mycoplasma } \\
\text { pneumoniae } \\
\text { pneumonia } \\
\text { (n=24) }\end{array}$ & $\begin{array}{l}\text { Non - Mycoplasma } \\
\text { pneumoniae } \\
\text { pneumonia } \\
\text { (n=142) }\end{array}$ \\
\hline Radiological characteristics & $12(50.0)$ & $83(58.4)$ \\
$\quad$ Linear opacities & $4(16.7)$ & $18(12.7)$ \\
Patchy infiltration & $2(8.3)$ & $22(15.5)$ \\
$\quad$ Segmental or lobar & $2(8.3)$ & $11(7.7)$ \\
consolidation & $2(8.3)$ & $6(4.2)$ \\
Interstitial infiltration & $2(8.3)$ & $2(1.4)$ \\
Reticulo-nodular infiltrate & 13.208 .28 & 16.8110 .51 \\
Pleural effusion & 69.3719 .35 & 68.9618 .60 \\
WBC (10 $/ L$, mean SD) & 24.0317 .25 & 24.8815 .66 \\
Neutrophils (\%) & 5.823 .50 & 4.652 .73 \\
Lymphocytis (\%) & 280.00 & 273.8398 .52 \\
Monocytes (\%) & 109.37 & \\
Platelet $\left(10^{9} / L\right.$, mean SD) & 51.7568 .29 & 90.9796 .92 \\
&
\end{tabular}

Date are presented as number (\%) unless otherwise indicated. WBC: white blood cell count; CRP: C-reactive protein; ${ }^{*} p<0.05$.

respectively). Furthermore, $58.3 \%$ children with MP pneumonia had radiological findings on the right side compared to $47.9 \%$ children with non-MP infection although the difference did not reach statistical significance $(p=0.418)$. We did not find a difference in total and differential WBC, platelet and CRP levels between study groups (Table 3 ). There were no significant associations between radiological or laboratory findings and MP infection as well.

Moreover, we analyzed group of children less than 5 years old and found that the proportion of MP pneumonia in this age group was $6.8 \%$. Interestingly, in children 5 years and older, $23.1 \%$ cases of CAP were caused by MP. Additionally, we compared clinical characteristics of children with MP pneumonia in these two groups and found that $66.7 \%$ of children below 5 years had coryza and $33.3 \%$ had difficulty breathing, while none of the older children had those symptoms $(\mathrm{p}=0.001, \mathrm{p}=0.011$, respectively). The younger children were more likely than the older children to have tachypnoea (33.3\% vs. 11.1\%: $\mathrm{p}=0.031$ ) as well. There were no other differences regarding clinical characteristics, radiological or laboratory findings when these two groups of children were compared.

Hospital admission between children with and without MP pneumonia showed no differences ( $91.7 \%$ vs. $88.7 \%$,

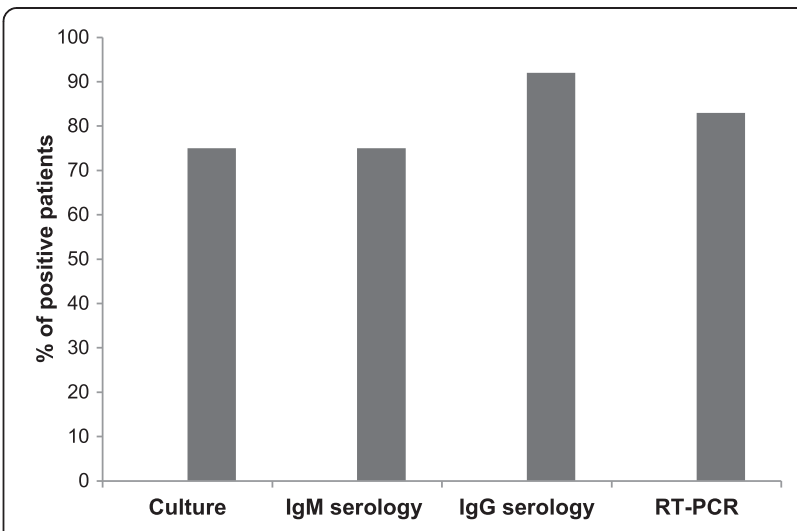

Figure 1 In a group of patients with Mycoplasma pneumoniae pneumonia, percents of positive patients for each method. RT-PCR: real-time polymerase chain reaction

$\mathrm{p}=0.669)$. However, children with MP infection had longer total duration of fever and cough compared to children with non-MP infection (6.33 3.73 vs. $4.683 .23, \mathrm{p}=0.02$; 16.045 .38 vs. $8.325 .51, \mathrm{p}=0.015$ respectively) Moreover children with MP pneumonia had longer duration of wheezing and length of hospitalization compared to children with non-MP pneumonia (2.25 3.33 vs. 0.591 .42 , $\mathrm{p}=0.001 ; 7.333 .40$ vs. $3.482 .78, \mathrm{p}=0.001$ respectively). None of children with MP pneumonia exhibited extrapulmonary manifestations. All children recovered without any of complication associated with MP infection.

Out of 24 children with MP pneumonia, 18 (75.0\%) were confirmed to be MP positive by serology, RT-PCR and culture, $4(16.7 \%)$ children were serologically positive only and 2 (8.3\%) were positive by RT-PCR only (Figure 1 ). Real-time PCR was positive in $81.8 \%$ serologically positive children. In all 20 RT-PCR positive children, the RT-PCR was performed 7.8 5.2 days after disease onset. A specific IgM antibody response in acute-phase sera was detected in 18 of $22(81.8 \%)$ serologically positive children whose

Table 4 Mycoplasma pneumoniae detection by IgM antibody testing, real-time polymerase chain reaction, culture and IgG antibody titers in paired sera

\begin{tabular}{llll}
\hline & & \multicolumn{2}{l}{$\begin{array}{l}\text { IgG antibody titers in paired } \\
\text { sera with a fourfold increase, }\end{array}$} \\
\cline { 3 - 4 } & & Positive & Negative \\
\hline IgM resulte & Positive & $18(10.84)$ & $0(0)$ \\
& Negative & $4(2.41)$ & $144(86.75)$ \\
RT-PCR results & Positive & $18(10.84)$ & $2(1.2)$ \\
& Negative & $4(2.41)$ & $142(85.54)$ \\
Culture results & Positive & $18(10.84)$ & $0(0)$ \\
& Negative & $4(2.41)$ & $144(86.75)$ \\
\hline
\end{tabular}

Date are presented as number (\%).

RT-PCR: real-time polymerase chain reaction. 
Table 5 Diagnostic values of different methods with a fourfold or greater increase of IgG antibody titers in paired sera as the gold standard

\begin{tabular}{lllll}
\hline & Sensitivity (\%) & Specificity (\%) & PPV (\%) & NPV (\%) \\
\hline Serology (IgM antibodies) & 81.82 & 100.00 & 100.00 & 97.30 \\
RT-PCR & 81.82 & 98.61 & 90.00 & 97.26 \\
Culture & 81.82 & 100.00 & 100.00 & 97.30 \\
\hline
\end{tabular}

Date are presented as percentage.

RT-PCR: real-time polymerase chain reaction; PPV: positive predictive value; NPV: negative predictive value; PLR: positive likelihood ratio.

acute-phase sera were taken 8.74 .8 days after disease onset. All 22 serologically positive children had fourfold increase in IgG antibody titers in convalescent-phase sera. The mean value of IgM and IgG were 1.651 .16 and 87.5840 .77 respectively. Moreover, we compared diagnostic value of three different methods, IgM serology in acute-phase sera, RT-PCR and culture, considering a fourfold or greater increase of IgG antibody titers in paired sera as the gold standard (Table 4). As shown in Table 5 sensitivity and specificity of all three methods were similar. On the contrary, when RT-PCR was considered as the gold standard IgM serology had the lowest sensitivity (Table 6). Additionally, we observed that combination of IgM detection in acute-phase serum and RT-PCR revealed positive results for 22 (91.7\%) of 24 cases with MP infection.

\section{Discussion}

In this study we assessed the incidence of MP pneumonia, analyzed clinical, radiological and laboratory characteristics of MP pneumonia and compared value of three different diagnostic methods.

We found that $14.5 \%$ cases of CAP in Serbia were caused by MP. Most hospital-based studies from Europe reported similar frequencies of MP pneumonia with rates ranging from $9 \%$ to $14 \%$ [6-11]. On the contrary, studies from Asia reported higher prevalence of MP pneumonia [12-15]. However, comparison with results obtained from other studies is difficult due to the heterogeneity of the epidemiologic conditions, studied population, diversity in clinical specimens, and diagnostic methods applied. Among MP positive children in present study, $75 \%$ were children between 515 years old. In accordance to our data various studies reported that children 5 years and older have a higher rate of MP infections compared to younger children $[6,9,10,16]$. We observed that there was no seasonal variation in MP infection which was also found in studies performed in Italy and Tunis $[17,18]$.

The clinical presentation of MP pneumonia observed in our study did not differ from those described in other studies $[9,10,14,19]$. One third of children with MP pneumonia had wheezing whereas fever and cough were the most common symptoms. When we compared clinical characteristics between children with MP pneumonia and non-MP pneumonia, we found that cough, headache and wheezing were more frequent in children with MP infection. Studies from Turkey and Brazil found that cough was contributing finding to MP infection $[16,20]$.

Higher incidence of wheezing in children with MP pneumonia in our study could be due to the fact that half of children with MP infection had asthma or atopy. However, children with non-MP pneumonia had high incidence of asthma or atopy as well (42\%) and no significant difference was found between this two groups. Moreover, logistic regression analysis showed that wheezing was significantly associated with MP pneumonia. Italian study found association between MP infection and wheezing in children 5 years old and over [21]. Furthermore, we observed that the radiological and laboratory findings did not distinguish MP pneumonia from other CAP as reported elsewhere $[10,14,17]$. Although the sample size of children less than 5 years old was small, we found that younger children more often had coryza, difficulty breathing and tachypnoea which is in agreement with previous reports $[9,22]$. The fact that all children below 5 years were hospitalized and probably had more severe disease could have contributed to this observation. Extrapulmonary manifestations were not observed in present study as previously reported [19].

Detection of IgM, in a single serum for diagnosis of MP infection is considered significant in children that

Table 6 Diagnostic values of different methods with a real-time polymerase chain reaction as the gold standard

\begin{tabular}{|c|c|c|c|c|c|}
\hline & Sensitivity (\%) & Specificity (\%) & PPV (\%) & NPV (\%) & PLR \\
\hline Serology (IgM antibodies) & 80.00 & 98.63 & 88.89 & 97.30 & 58.40 \\
\hline Serology (fourfold increase lgG antibodies) & 90.00 & 97.26 & 81.82 & 98.61 & 32.85 \\
\hline Culture & 90.00 & 100.00 & 100.00 & 98.65 & \\
\hline
\end{tabular}

Date are presented as percentage.

RT-PCR: real-time polymerase chain reaction; PPV: positive predictive value; NPV: negative predictive value; PLR: positive likelihood ratio. 
had fewer opportunities for repeated exposure [23]. The sensitivity and specificity of IgM serology in present study were $81.82 \%$, and $100 \%$ respectively compared to IgG serology and $80.00 \%$ and $98.63 \%$ respectively compared to RT-PCR. Nadal et al. reported sensitivity and specificity of IgM serology related to serology of $78.1 \%$ and $87.1 \%$ respectively in children with MP pneumonia [7]. Chang et al. used RT-PCR as a reference method and observed that sensitivity and specificity of IgM serology were $62.2 \%$ and $85.5 \%$ respectively [24]. Sensitivity of serology could be affected by timing of specimen collection. This could explain high sensitivity of IgM serology in present study, considering that sera were taken in the second week after disease onset. According to the literature detection of IgG antibodies in paired sera is more sensitive method, which was also confirmed by our results [25].

Our results show that sensitivity and specificity of RTPCR compared to IgG serology were $81.82 \%$ and $98.61 \%$ respectively. Nadal et al. found similar sensitivity and specificity of RT-PCR compared to serology in children with MP pneumonia [7]. Morozumi et al. reported higher sensitivity and similar specificity of RT-PCR related to serology, $90.2 \%$ and $97.9 \%$ respectively [26]. However, some recent studies report lower sensitivity and specificity of this method compared to IgM serology [24,27] and compared to IgG serology $[28,29]$. This heterogeneity between studies regarding sensitivity of PCR method could be due to the different PCR types, different gene targets or different sample type and time point for sampling [30]. According to the literature RT-PCR revealed higher sensitivity compared to others types of PCR [30,31], but this could only partially explain our results. Finally, we found that combination of IgM detection in acute-phase serum and RT-PCR was positive for $91.7 \%$ cases in children with MP infection indicating a possible use of both techniques as a valid diagnostic approach in early detection of MP infection in children with CAP.

However, present study showed some discrepancies between the results of the diagnostic methods used. The 4 cases of negative RT-PCR results with positive serology may be due a disappearance, or a low level of MP load in the sample [25]. The 2 positive RT-PCR results in serologically negative children may be due to an inadequate immune response or detection of a carrier state [32].

Our study had some limitations. One of them is the small sample size. Besides, the results might be limited due to the fact that children in this study were presenting to a tertiary children hospital, therefore patients with more severe infections may have been overrepresented. Furthermore, we did not perform extensive microbiological testing, therefore we cannot exclude the possibility that some children might have had co-infections with other bacterial or viral pathogens.

\section{Conclusions}

The present study has shown that there are no characteristic radiological findings, or routine laboratory tests that would distinguish CAP caused by MP from other CAP. Our results suggest that some clinical features are indicative for MP pneumonia, such as headache and wheezing and therefore may aid the process differentiating it from pneumonia cases caused by other pathogens. However, detection of IgM antibodies together with RTPCR allows for precise and reliable diagnosis of MP infections in children during the acute phases of disease, indicating a possible use of both techniques as a valid diagnostic approach in early detection of MP infection in children with CAP.

\section{Competing interests}

The authors declare that they have no competing interests.

\section{Authors contributions}

All the authors contributed equally to study conception, data acquisitions and analysis, drafting of the manuscript and approval of the final version of the manuscript.

\section{Acknowledgements}

This research was financially supported by the ministry of Science and Technological Development of the Republic of Serbia. Project No.175039.

\section{Author details}

Medical Faculty, University of Belgrade, Dr Subotica 8, 11000 Belgrade, Serbia. ${ }^{2}$ University Children s Hospital, Tirsova 10, Belgrade, Serbia. ${ }^{3}$ Children s Hospital for Respiratory Diseases and TB, Clinical Health Center Dr Dragisa Misovic - Dedinje, Jovana Marinovica 4, 11000 Belgrade, Serbia. ${ }^{4}$ Institute of Microbiology, Medical Faculty, University of Belgrade, Dr Subotica 8, 11000 Belgrade, Serbia.

Received: 4 November 2014 Accepted: 9 December 2014

Published online: 18 December 2014

\section{References}

1. Waites K, Talkington D: Mycoplasma pneumoniae and Its Role as a Human Pathogen. Clin Microbiol Rev 2004, 17:697 728.

2. Harris M, Clark J, Coote N, Fletcher P, Harden A, McKean M, Thomson A, British Thoracic Society Standards of Care Committee: British Thoracic Society guidelines for the management of community acquired pneumonia in children: update 2011. Thorax 2011, 66:1 23.

3. Loens K, Ursi D, Goossens H, Leven M: Molecular diagnosis of Mycoplasma pneumoniae respiratory tract infections. J Clin Microbiol 2003, 41:4915 4923.

4. Mclntosh K: Community-acquired pneumonia in children. $N$ Engl J Med 2002, 346:429 437.

5. Davies H, Wang E, Manson D, Babyn P, Shuckett B: Reliability of the chest radiograph in the diagnosis of lower respiratory infections in young children. Pediatr Infect Dis J 1996, 15:600 604.

6. Waris ME, Toikka P, Saarinen T, Nikkari S, Meurman O, Vainionpaa R, Mertsola J, Ruuskanen O: Diagnosis of Mycoplasma pneumoniae pneumonia in children. J Clin Microbiol 1998, 36:3155 3159.

7. Nadal D, Bossart W, Zucol F, Steiner F, Berger C, Lips U, Altwegg M: Community-acquired pneumonia in children due to Mycoplasma pneumoniae: diagnostic performance of seminested 16S rDNA-PCR. Diagn Microbiol Infect Dis 2001, 39:15 19

8. Puljiz I, Kuzman I, Dakovic-Rode O, Schnwald N, Mise B: Chlamydia pneumoniae and Mycoplasma pneumoniae pneumonia: comparison of clinical, epidemiological characteristics and laboratory profiles. Epidemiol Infect 2006, 134:548 555.

9. Defilippi A, Silvestri M, Tacchella A, Giacchino R, Melioli G, Di Marco E, Cirillo C, Di Pietro P, Rossi GA: Epidemiology and clinical features of Mycoplasma pneumoniae infection in children. Respir Med 2008, 2:1762 1768. 
10. Almasri M, Diza E, Papa A, Eboriadou M, Souliou E: Mycoplasma pneumoniae respiratory tract infections among Greek children. Hippokratia 2011, 15:147 152

11. Kicinski $\mathrm{P}$, Wisniewska-Ligier M, Wozniakowska-Gesicka T: Pneumonia caused by Mycoplasma pneumoniae and Chlamydophila pneumoniae in children - comparative analysis of clinical picture. Adv Med Sci 2011 56:56 63.

12. Kashyap B, Kumar S, Sethi GR, Das BC, Saigal SR: Comparison of PCR, culture \& serological tests for the diagnosis of Mycoplasma pneumoniae in community-acquired lower respiratory tract infections in children. Indian J Med Res 2008, 128:134 139.

13. Liu FC, Chen PY, Huang FL, Tsai CR, Lee CY, Wang LC: Rapid diagnosis of Mycoplasma pneumoniae infection in children by polymerase chain reaction. J Microbiol Immunol Infect 2007, 40:507 512.

14. Samransamruajkit R, Jitchaiwat S, Wachirapaes W, Deerojanawong J, Sritippayawan S, Prapphal N: Prevalence of Mycoplasma and Chlamydia pneumonia in severe community-acquired pneumonia among hospitalized children in Thailand. Jpn J Infect Dis 2008, 61:36 39.

15. Liu CL, Wang GQ, Zhang B, Xu H, Hu LP, He XF, Wang JH, Zhang JH, Liu XY, Wei M, Liu ZY: Mycoplasma pneumoniae pneumonia in hospitalized children diagnosed at acute stage by paired sera. Chin Med J 2010, 123:3444 3450.

16. Sidal M, Kilic A, Unuvar E, Oguz F, Onel M, Agacifidan A, Aydin D, Koksalan K, Beka $\mathrm{H}$ : Frequency of Chlamydia pneumoniae and Mycoplasma pneumoniae infections in children. J Trop Pediat 2007, 53:225 231.

17. Esposito S, Blasi F, Bellini F, Allergra L, Principi N and the Mogwil study group: Mycoplasma pneumoniae and Chlamydia pneumoniae infections in children with pneumonia. Eur Respir J 2001, 17:241 245.

18. Touati A, Pereyre S, Bouziri A, Achour W, Khaldi A, Ben Jaballah N, Bbar C, Ben Hassen A: Prevalence of Mycoplasma pneumoniae-associated respiratory tract infections in hospitalized children: results of a 4-year prospective study in Tunis. Diagn Microbiol Infect Dis 2010, 68:103 109.

19. Hsieh SC, Kuo YT, Chern MS, Chen CY, Chan WP, Yu C: Mycoplasma pneumonia: clinical and radiographic features in 39 children. Pediatr Int 2007, 49:363 367

20. Vervloet LA, Camargos PA, Soares DR, Oliveira GA, Oliveira JN: Clinical, radiographic and hematological characteristics of Mycoplasma pneumoniae pneumonia. J Pediatr 2010, 86:480 487.

21. Esposito S, Blasi F, Arosio C, Fioravanti L, Fagetti L, Droghetti R, Tarsia P, Allegra L, Principi N: Importance of acute Mycoplasma pneumoniae and Chlamydia pneumoniae infections in children with wheezing. Eur Respir J 2000, 16:1142 1146 .

22. Othman N, Isaacs D, Kesson A: Mycoplasma pneumoniae infections in Australian children. J Paediatr Child Health 2005, 41:671 676

23. Waites KB, Thacker WL, Talkington DF: The value of culture and serology for detection of Mycoplasma pneumoniae infections in the clinical laboratory in the age of molecular diagnosis. Clin Microbiol Newsletter 2001, 23:123 129.

24. Chang HY, Chang LY, Shao PL, Lee PI, Chen JM, Lee CY, Lu CY, Huang LM: Comparison of real-time polymerase chain reaction and serological tests for the confirmation of Mycoplasma pneumoniae infection in children with clinical diagnosis of atypical pneumonia. J Microbiol Immunol Infect 2014, 47:137 144.

25. Atkinson TP, Balish MF, Waites KB: Epidemiology, clinical manifestations, pathogenesis and laboratory detection of Mycoplasma pneumoniae infections. FEMS Microbiol Rev 2008, 32:956 973.

26. Morozumi M, Nakayama E, Iwata S, Aoki Y, Hasegawa K, Kobayashi R, Chiba N, Tajima T, Ubukata K: Simultaneous detection of pathogens in clinical samples from patients with community-acquired pneumonia by real-time PCR with pathogen-specific molecular beacon probes. J Clin Microbio/ 2006, 44:1440 1446

27. Chaudhry R, Sharma S, Javed S, Passi K, Dey AB, Malhotra P: Molecular detection of Mycoplasma pneumoniae by quantitative real-time PCR in patients with community acquired pneumonia. Indian J Med Res 2013, 138:244 251.

28. Qu J, Gu L, Wu J, Dong J, Pu Z, Gao Y, Hu M, Zhang Y, Gao F, Cao B, Wang C, Beijing Network for Adult Community-Acquired Pneumonia (BNACAP): Accuracy of IgM antibody testing, FQ-PCR and culture in laboratory diagnosis of acute infection by Mycoplasma pneumoniae in adults and adolescents with community-acquired pneumonia. BMC Infect Dis 2013, 13:172.

29. Martnez MA, Ruiz M, Zunino E, Luchsinger V, Avendao LF: Detection of Mycoplasma pneumoniae in adult community-acquired pneumonia by PCR and serology. J Med Microbiol 2008, 57:1491 1495
30. Zhang L, Zong ZY, Liu YB, Ye H, LV XJ: PCR versus serology for diagnosing Mycoplasma pneumoniae infection: a systematic review \& meta-analysis. Indian J Med Res 2011, 134:270 280.

31. Daxboeck F, Khanakah G, Bauer C, Stadler M, Hoffmann H, Stanek G: Detection of Mycoplasma pneumoniae in serum specimens from patients with Mycoplasma pneumoniae by PCR. Inter J Med Microbiol 2005 , 295:279 285.

32. Waites KB, Balish MF, Atkinson TP: New insights into the pathogenesis and detection of Mycoplasma pneumoniae infections. Future Microbiol 2008 3:635 648.

doi:10.1186/s13052-014-0104-4

Cite this article as: Medjo et al:: Mycoplasma pneumoniae as a causative agent of community-acquired pneumonia in children: clinical features and laboratory diagnosis. Italian Journal of Pediatrics 2014 40:104.

\section{Submit your next manuscript to BioMed Central and take full advantage of:}

$\otimes$ Convenient online submission

$\otimes$ Thorough peer review

$\nabla$ No space constraints or color $\nabla$ gure charges

$\otimes I m m e d i a t e$ publication on acceptance

$\triangle$ Inclusion in PubMed, CAS, Scopus and Google Scholar

$\otimes$ Research which is freely available for redistribution

Submit your manuscript at www.biomedcentral.com/submit
C Biomed Central 